\title{
The Effect of Therapeutic Low-Frequency Ultrasound applied to Myofascial Trigger Points: A Pilot Pre-Post Design Study
}

\author{
Michal Elboim Gabyzon ${ }^{1 *}$, Amit Drat ${ }^{2}$, Leonid Kalichman ${ }^{2}$ \\ ${ }^{1}$ Physical Therapy Department, Faculty of Social Welfare and Health Sciences, University of Haifa, Haifa, Israel. \\ ${ }^{2}$ Department of Physical Therapy, Faculty of Health Studies, Ben Gurion University of the Negev, Beer Sheva, Israel.
}

Received 28 February 2021; Revised 14 May 2021; Accepted 17 June 2021; Published 01 September 2021

\begin{abstract}
Ultrasound at a frequency of 1 or $3 \mathrm{MHz}$ is frequently used to treat various musculoskeletal conditions, but research on ultrasound operating at $38-50 \mathrm{kHz}$ frequencies (US-KHz) is lacking. Study aimed to evaluate the short-term effect of US$\mathrm{KHz}$ on pain pressure threshold (PPT), ankle dorsiflexion range of motion (ROM), and motor performance (the Side Hop Test) in subjects with a myofascial trigger point $(\mathrm{TrP})$ in the calf muscle. US-KHz was applied to the area of the palpable TrP in the calf muscle for 10 minutes (continuous pulse, transducer head size $19.6 \mathrm{~cm}^{2}$, power $0.75-1.25 \mathrm{w} / \mathrm{cm}^{2}$ ) in twenty volunteers $(18-45$ years old). Significant improvements $(\mathrm{p}<0.001)$ were noted in the Side Hop Test 24 hours after the intervention. ROM improved significantly after 5 minutes with changes maintained 24 hours later. No change was found in the PPT. This pilot study presents preliminary evidence of the efficacy of US-KHz in treating TrPs.
\end{abstract}

Keywords: Therapeutic Ultrasound; Kilo-Herz Frequency; Trigger Points; Feasibility Study; Calf Muscles.

\section{Introduction}

Myofascial pain is a common pain syndrome of the skeletal muscles, frequently accompanied by myofascial trigger points (TrPs). These TrPs are sensitive spots found within the muscle and are generally identified by local muscle palpation $[1,2]$. Although there is much debate amongst researchers and clinicians as to the diagnostic criteria of $\operatorname{TrPs}$, a recent international Delphi panel concurred that a diagnosis is rendered when two of the following three criteria are met: a hypersensitive spot within a muscle which may include in addition to a sharp pain, sensations such as a dull ache, or tingling; a taut muscle band; and a referred sensation extending to distant sites [2,3]. TrPs are usually classified as either active or latent. The local and referred symptoms of active TrPs are spontaneous and increase when manipulated. In contrast, while latent TrPs are not associated with spontaneous discomfort, local and referred symptoms may be provoked by mechanical stimulation, such as finger pressure exerted over the latent TrPs [4]. Similar to active $\operatorname{TrPs}$, latent TrPs may restrict the range of motion (ROM) and may lead to muscle dysfunction, i.e., muscle weakness, muscle imbalance, or altered motor unit recruitment patterns $[5,6]$.

The etiology of myofascial pain and TrPs is not as yet fully understood. Treatment is usually symptomatic with the intent to reduce pain and improve ROM and muscle strength [4]. Diverse invasive and non-invasive modalities have been reported with conflicting results. These modalities include local injections, dry needling, deep tissue massage, stretching spray techniques, laser therapy, electrotherapy, and superficial and deep heat [7-9]. Therapeutic ultrasound (US) is a commonly used modality applied to treat myofascial pain [10]. The US transforms electrical energy into

* Corresponding author: michal.elboim@gmail.com

http://dx.doi.org/10.28991/SciMedJ-2021-0303-5

$>$ This is an open access article under the CC-BY license (https://creativecommons.org/licenses/by/4.0/).

(C) Authors retain all copyrights. 
mechanical oscillation via piezoelectric crystals [11]. The oscillation of particles within the biological tissues, due to the longitudinal wave created by the US, results in an increase in deep tissue temperature and mechanical modifications at the cellular level [12], thereby, possibly modulating pain, increasing tissue elasticity and ROM, improving blood flow, reducing muscle spasms, and enhancing the tissue healing process.

The efficacy of using US to treat TrPs has been investigated in several studies culminating with conflicting results. A recent systematic review and meta-analysis of randomized controlled studies identified ten studies that assessed the therapeutic effects of US on myofascial pain [13]. The review indicated that US treatment significantly reduces pain intensity with no concurrent significant effect on ROM. The authors concluded that due to the high risk of bias and the significant heterogeneity of the studies in terms of treatment protocols and dosage, the results of the meta-analysis did not clearly support the use of US as an effective method to treat myofascial pain. The amount of US energy delivered and absorbed by the biological tissues depends on a variety of dosage parameters, i.e., pulse frequency, intensity, and duration of application [14-16]. Typical frequencies of conventional US range between 1-3 MHz, with the higher frequencies associated with a lower penetration power [17]. It has been suggested that much lower frequencies (associated with longer wavelengths) would provide a more divergent field shape. Furthermore, reducing the frequency would result in less attenuation of the sonic energy as it travels inward. There would also be a more uniform distribution of the absorbed energy in the direction of wave propagation, thus, expecting greater thermal and athermal effects in the deeper tissues [18]. Due to these properties, there is a growing interest in applying US at $\mathrm{kHz}$ frequencies.

Despite the theoretical advantages of low-frequency US, only a few studies have investigated its efficacy in clinical settings. Several studies have focused on soft tissue healing. A systematic review and meta-analysis of eight randomized controlled trials revealed that low-frequency US at both low and high intensities may accelerate wound healing in patients with venous stasis and diabetic foot ulcers [19]. Fewer research studies relating to musculoskeletal impairments have been conducted, resulting in conflicting outcomes. Thus, in a prospective randomized trial comparing the efficacy of US at $3 \mathrm{MHz}$ with that of $45 \mathrm{KHz}$ in the treatment of acute ankle sprains, it was determined that the group treated with a lower US frequency demonstrated greater immediate improvements in gait parameters, remaining statistically significant three days following treatment [18]. In contrast, Meakins and Watson (2006) [19] found that low-frequency US is as effective as hot packs in increasing functional ankle mobility. In light of these conflicting results, the goal of this pilot study was to examine the immediate and short-term effects of KHZ-frequency US on pain pressure threshold (PPT), ankle dorsiflexion ROM, and motor performance (Single Leg Hop Test) in subjects with latent TrPs located in the calf muscle.

\section{Materials and Methods}

\subsection{Design}

A pre-post design pilot interventional study.

\subsection{Setting}

Department of Physical Therapy, Recanati School for Community Health Professions, Faculty of Health Sciences, Ben-Gurion University of the Negev, Beer Sheva, Israel.

\subsection{Sample}

The study sample included 20 healthy students attending the Ben-Gurion University of the Negev, between the ages of 18-45. The trial was advertised through an ad posted on the university's bulletin board and social networks. Inclusion criteria consisted of the presence of at least one TrP located in the calf muscle of one leg, which was identified by manual palpation by a tester. If more than one TrP was found, the one with a lower PPT was used. Exclusion criteria included a background of neurological diseases, active systemic diseases affecting sensation or perception of pain (i.e., diabetes, peripheral vascular disease, cancer, rheumatoid arthritis, osteoarthritis), a fracture or ankle sprain during the six months prior to the study, pregnancy, local infection or inflammation, and contraindications to US (malignant tumors, active bleeding or open wound in the calf area, and systemic vascular disease).

\subsection{Intervention}

Each participant underwent a single US application of a 38-kHz frequency (SIRIO, BAC Technology, Italy), head size $19.6 \mathrm{~cm}^{2}$, in a continuous current according to the manufacturer's instructions. A standard water-based US gel was used. The maximum power of the device was $2.5 \mathrm{~W} / \mathrm{cm}^{2}$. The intervention was performed by a physical therapy student trained in operating the device. The participant was informed prior to treatment that he would not feel any abnormal sensations such as strong heat or pain but might feel a buzzing in his ears and a low-medium heat sensation in the placement area. The treatment procedure was performed on the area of the marked point (a dot was drawn on the skin with a marker by the examiner at the initial examination, thereby, accurately mapping the TrP). The procedure lasted 10 
minutes, commencing at a $30 \%$ maximum intensity, subsequently increased to $40 \%$ after 2 minutes of treatment. If the participant was still feeling comfortable, intensity was increased to 50\%. After an additional 2 minutes, if the patient reaffirmed that he/she still felt comfortable, the therapist raised the intensity again. If after increasing the intensity, the patient reported discomfort or pain, the therapist lowered the intensity back to the previous intensity. At any stage throughout the treatment, the patient could ask to lower the intensity if he/she felt uncomfortable or in pain. We chose this regimen because in our preliminary stage when we tested different parameters of US (we aimed to apply the US at $50 \%$ of the maximum intensity), some subjects reported that $50 \%$ felt too hot.

\subsection{Outcome Measures}

All outcome measures were documented by a single researcher (a 4th-year physical therapy student), who had undergone intensive specific training in evaluation techniques. Demographics were collected using a questionnaire and included sex, weight, height, smoking, and background diseases. Body mass index (BMI) was calculated as weight $(\mathrm{kg}) /$ height $\left.(\mathrm{m})^{2}\right)$.

\subsubsection{Weight-Bearing Lunge Test (WBLT)}

This test was used to assess ankle dorsiflexion ROM. The WBLT has been used to detect ROM deficits in individuals with chronic ankle instability [20] and to track the progress of ROM improvement during the rehabilitation [21]. Hall and Docherty (2017) [22] found that WBLT highly correlates with measurements performed using a 2D motion capture system and determined that WBLT is a valid and reliable tool to assess dorsiflexion ROM. Furthermore, Bennell et al. found that the WBLT demonstrated excellent inter and intra-rater reliability [23]. Another study found that the WBLT exhibited high test-retest reliability (ICC $=0.93-0.99)[24]$.

We assessed the dorsiflexion ROM angle between the anterior border of the tibia and the vertical line using a digital inclinometer. The examiner used a non-permanent felt-tipped pen to mark a dot on the anterior border of the tibia, $15 \mathrm{~cm}$ below the tibial tuberosity of both legs. The test procedure was demonstrated to the subjects together with standardized instructions before the commencement of the procedure. The subject then positioned the tested foot so that the heel line and the big toe were aligned on white masking tape. With both feet stationary, a controlled forward lunge was performed, thereby, flexing the knee as the subject attempted to reach maximal dorsiflexion. Pronation or supination of the foot was not controlled. During the tests, subjects were allowed to hold onto the wall for balance. At the maximum lunge point, the examiner placed the inclinometer on the tibial mark and recorded the achieved angle. Two additional measurements were taken after the subject stood and resumed a comfortable position. The best result was recorded by the examiner.

\subsubsection{Side Hop Test}

This test assessed functional performance ability and dynamic impairments. The gold standard for muscle strength evaluation is the isokinetic muscle torque test [25], however, its validity has been questioned due to the low correlation between the isokinetic muscle torque and functional performance [26]. It has been suggested to use dynamic muscle power tests to evaluate muscle function [27] given that the ability to produce high forces during high velocities is one of the most important factors in sports performances [28]. In addition to power tests, hop tests have been highly recommended to evaluate sport-specific performances in healthy athletes and after various interventions [29, 30]. A battery of hop tests evaluating hop performances has been previously described and analyzed for reliability [31].

There are three tests with a high capacity to discriminate hop performance: the vertical jump, the hop for distance, and the side hop. For the present study, the Side Hop Test was employed. Reliability was conducted for this test and determined to be good with an ICC of 0.84 [27, 32]. Docherty et al. method was used in this study [33]. All subjects were instructed to hop laterally on the right limb a $30 \mathrm{~cm}$ distance and then return to the starting location. Subjects were told that the goal was to complete ten repetitions as quickly as possible. If a subject fell, or put the contralateral foot down, or did not completely clear the $30 \mathrm{~cm}$ distance while hopping, the trial was regarded as unacceptable and the subject had to repeat the task. Subjects were familiarized with the task by completing 3-4 repetitions at partial speed. Following a rest period, they completed their first trial, rested for at least 60 seconds, then completed the second. The examiner recorded the time with a handheld stopwatch to the nearest hundredth of a second. The best trial (shortest) was used for analysis.

\subsubsection{Pain Pressure Threshold}

PPT of the identified TrP assessed the latent TrP pressure sensitivity using a mechanical algometer. The disc was placed perpendicularly on the TrP. The examiner then increased the intensity of pressure until the participant reported initial feelings of pain. The score was determined by averaging three consecutive measurements. The algometer was found to be valid and reliable in repeated measurements (Interrater, Intrarater) in normal muscles in healthy people with a minimal clinically important difference of 14.71-19.61 N/cm² [34]. Chesterton et al. [35] found high reliability when three repeated measures were averaged: $\mathrm{ICC}=0.91(\mathrm{CI} 0.82 ; 0.9795 \%)$ with a minimal clinically important difference 
of $17.39 \mathrm{~N} / \mathrm{cm}^{2}$. All outcome measure evaluations were performed prior to the intervention, five minutes after the intervention, and 24 hours after the intervention.

\subsection{Research Procedure}

The subjects chosen in accordance with the exclusion and inclusion criteria were informed of the experimental procedure. After signing an informed consent form, the examiner palpated the participant's calf muscles while the subject lay on his/her stomach with a slightly bent knee. If a TrP was detected the examiner marked the TrP as described above. After completing the demographic questionnaire, the three tests were performed under the examiner's guidance in the following order: PPT, ankle dorsiflexion ROM, and Side Hop Test. The subject was asked to lay prone, and the intervention was performed according to the above-detailed protocol. Five minutes after the intervention ended, PPT and ankle dorsiflexion ROM evaluations were repeated. Approximately 24 hours after the intervention, the patient was asked to return and perform an additional set of all three tests.

\subsection{Statistical Analyses}

All statistical analyses were performed using the SPSS statistical package (version 23 for Windows). Significance levels were set at $\mathrm{P}<0.05$. Descriptive statistics characterized the sample. The comparisons between the background, 5 minute post-intervention, and 24-hour post-intervention measurements were calculated by the repeated measurements ANOVA using a posthoc analysis. A Pearson correlation analysis assessed the association between changes in the outcome measures (delta scores=24-hour post-intervention measurement minus the baseline measurement).

\section{Results}

The study included 20 subjects (19 females, one male) ranging in age from $24-32$, the mean age of $26.65 \pm 2.78$. The mean BMI was $21.90 \pm 2.84 \mathrm{~kg} / \mathrm{m}^{2}$; most (85\%) reported routinely exercising. Additional demographic characteristics are presented in Table 1.

Table 1. Descriptive statistics $(\mathrm{N}=20)$

\begin{tabular}{cc}
\hline Variables & Mean \pm SD \\
\hline Age (years) & $26.65 \pm 2.78$ \\
Weight $(\mathrm{kg})$ & $60.30 \pm 7.97$ \\
Height $(\mathrm{m})$ & $1.66 \pm 0.06$ \\
BMI $\left(\mathrm{kg} / \mathrm{m}^{2}\right)$ & $21.90 \pm 2.84$ \\
\hline Sex (females) & $\mathbf{N}(\%)$ \\
Smoking & $19(95 \%)$ \\
Regular physical activity & $1(5 \%)$ \\
\hline
\end{tabular}

SD: standard deviation; BMI: body mass index

The main results are summarized in Table 2 .

Table 2. Comparison (results of repeated measurements ANOVA) of measurements before and after the intervention

\begin{tabular}{ccccc}
\hline Variable & $\begin{array}{c}\text { Before } \\
\text { Mean } \pm \text { SD }\end{array}$ & $\begin{array}{c}\text { 5 min. after } \\
\text { Mean } \pm \text { SD }\end{array}$ & $\begin{array}{c}\text { 24 hours after } \\
\text { Mean } \pm \text { SD }\end{array}$ & Comparison \\
PPT $\left(\mathrm{kg} / \mathrm{cm}^{2}\right)$ & $0.22 \pm 0.552$ & $0.44 \pm 0.712$ & $0.32 \pm 0.782$ & $\mathrm{~F}=0.817, \mathrm{p}=0.439$ \\
WBLT $(0)$ & $137.30 \pm 7.41$ & $139.50 \pm 7.69$ & $140.10 \pm 7.55$ & $\mathrm{~F}=8.767, \mathrm{p}<0.001 *$ \\
Side Hop Test $(\mathrm{sec})$ & $28.54 \pm 22.34$ & & $21.47 \pm 16.17 \quad \mathrm{~F}=15.027, \mathrm{p}<0.001 \quad$ \\
\hline
\end{tabular}

SD: standard deviation; PPT: pressure pain threshold; WBLT: weight-bearing lunge test;

Statistically significant $(\mathrm{p}<0.05)$ differences are marked in bold.

* In a pairwise comparison, significant differences were found between the 1st and 2nd measurements, and the 1st and 3rd measurements, but not between the 2 nd and 3rd measurements.

The PPT showed some increase after the US application between the 1st (performed before placement) and the 2nd test (five minutes after placement), and between the 1 st and 3rd test ( 24 hours after placement), although, this change was not statistically insignificant $(\mathrm{P}=0.439)$. 
In the ankle dorsiflexion ROM tested by the WBLT, an average improvement of $\sim 2^{\circ}$ between the 1 st and 2 nd test and an improvement of $\sim 1^{\circ}$ between the 2 nd and 3rd test was observed. The differences between the 1 st and 2 nd test and between the 1 st and 3rd test were statistically significant $(\mathrm{p}<0.001)$, however, no significant difference was found between the 2nd and 3rd test. Regarding motor performance tested by the Side Hop Test, a statistically significant difference was found between the 1 st and 2 nd tests $(\mathrm{p}<0.001)$. The mean difference between the 1 st and 2 nd test was 7.07 seconds (that is higher than a minimally detectable change of 2.8-5.7 seconds [36]). The Pearson correlation analysis assessed the relationship between changes in the measurement results; the only significant correlation $(r=-0.443$, $p=$ 0.05) was found between changes in the PPT (five minutes after treatment) and changes in the Side Hop Test. Subjects who further improved in the PPT during the 2nd test also improved in motor performance in the 2nd test (24 hours after placement).

\section{Discussion}

We determined that a single application of US-KHz directed at latent TrPs located in the calf muscles of healthy subjects results in an immediate increase in the ROM lasting 24 hours after the intervention. We also discovered that the US-KHz led to improvements in the Side Hop Test 24 hours following the intervention, however, no effect on PPT was found. To the best of our knowledge, only three previously published studies have examined the effect of US-KHz applied to musculoskeletal structures in vivo. The earliest study, a randomized placebo-controlled work published in 1996 compared the immediate effects of a single treatment with low-frequency US (45-KHz) to treatment with US at a high frequency (3- MHz), following an acute ankle sprain. Significant greater improvements in gait parameters following the lower frequency US were observed. The authors attributed this to a greater analgesic effect [18]. In contrast, Basso and Pike's [37] double-blinded study of subjects with a post-Colles fracture, found no difference in wrist ROM between patients treated with $46.39 \mathrm{US}-\mathrm{KHz}$ and sham treatments. However, it appears that the subjects were exposed to only one treatment and were examined two and eight weeks later; Thus, many confounding variables could have affected the results. The treatment and assessment protocol of the present study has more in common with the more recently published study by Meakins and Watson (2006) [19] who examined the effects of US-KHz on ankle ROM in non-injured individuals. Similar to our study, their results demonstrated statistically significant increases in ankle mobility following the application of US-KHz compared to the controls, nonetheless, they found that US-KHz was just as effective as hot packs in increasing ankle mobility.

Meakins and Watson's treatment protocol was based on Ward and Robertson [38] who applied US-KHz to non-living pig tissue demonstrating a 4-50C increase in superficial tissue temperature following 5-6 minutes of treatment. When examining the ROM of the human ankle, the authors increased the duration time by 4 minutes to compensate for the circulation's cooling effect and the moving treatment head. As increased tissue temperature has been shown to enhance connective tissue extensibility, as well as altering nerve conduction velocity, and increasing blood flow [11, 39], the authors considered the increase in ankle ROM as the result of enhanced tissue extensibility due to increased tissue temperature.

Dosage parameters in the present study were based on the manufacturer's recommendations (SIRIO, BAC Technology, Italy). While these parameters were not identical to those presented by Meakins and Watson, they were quite similar, i.e., treatment duration was 14 minutes in the present study, vs. 10 minutes in their study. Intensity ranged between $0.75-1.25 \mathrm{~W} / \mathrm{cm}^{2}$ in the present study vs. $0.94 \mathrm{~W} / \mathrm{cm}^{2}$ in their study. Hence, it appears that the same mechanism, i.e., increased tissue temperature, was responsible for the positive treatment effect on ankle ROM observed here as well. As latent TrP causes muscle dysfunction [6, 39], the thermal effect of the US-KHz might also explain the demonstrated improvement in the Side Hop Test [6]. Previous studies have shown positive effects of US-MHz frequencies on latent and active TrP in terms of ROM, PPT, pain relief, disability, and quality of life [11,40-44], however, no studies have as yet compared the effects of low frequency $(\mathrm{KHz})$ and high frequency $(\mathrm{MHz})$ US on TrPs. Based on the promising preliminary results of the current pilot feasibility study, it is essential to expand these results in follow-up larger controlled studies which will include comparisons between the effectiveness of US-KHz with the 'traditional' US-MHz in the treatment of TrPs.

In previous studies investigating MHZ frequency US, a significant beneficial effect on PPT indices of participants with soft tissue injury was observed [11, 40-42]. The gap found in these indices between the MHZ frequency studies and this study may lie in the deep penetration potential of the KHZ frequency, which may be 20 times greater than the MHZ frequency. We, therefore, feel that a different and more subtle treatment protocol should be adopted due to the great power of the device.

We observed that some people were more sensitive than others and reported discomfort or pain during the intervention. For these individuals, the intervention continued until the end of the allotted time, but the US intensity was lowered to a level that was comfortable for the participant. Our impression was that people with greater sensitivity to treatment showed less improvement in terms of pain pressure index in post-treatment tests. It may be worthwhile to perform a sensitivity test before applying the treatment as part of the research protocol in order to monitor people with 
skin sensitivity. Further studies should concentrate on the applied muscle, as previous studies have suggested that certain muscles are more easily influenced by superficial heating, resulting in an immediate change in muscle length and flexibility, for example, shoulder external rotator muscles were more affected by superficial heating than the triceps surae [39].

This study has several major limitations. Firstly, there was no control group; all subjects underwent the same treatment in the same way. This study design does not allow for controlling potential biases, i.e., a placebo effect or spontaneous change in outcomes (time effect). Moreover, a pre-post-designed study does not allow the blinding of tester or subjects. However, as mentioned before, this is a pilot, feasibility study performed as a preliminary stage to ascertain if US-KHz causes any change in subjects' outcomes, in addition to serving as a basis for a full-scale clinical trial.

\section{Conclusion}

The current results support the potential application of US-KHz therapy as an effective treatment modality for TrPs, by affecting elasticity and muscle flexibility (represented in this study by ROM) and improving muscle performance of the muscle. If this efficacy will be confirmed in a further full-scale clinical trial, US-KHz can be a therapeutic alternative to invasive treatments such as dry needling in TrP conditions and can be added to the physical therapist's toolbox. US$\mathrm{KHz}$ is a relatively new modality and only a few studies have examined its effect, therefore, high-quality, especially randomized controlled trials, are essential to establish its efficacy and treatment protocols. Further studies should also examine the effectiveness of this treatment on active TrPs as well as on TrPs in other areas of the body.

\section{Declarations}

\subsection{Author Contributions}

Conceptualization, M.E.G and L.K.; methodology, M.E.G and L.K.; formal analysis, M.E.G and L.K.; data curation, A.D and L.K; writing - M.E.G A. D and L.K; writing - review and editing, M.E.G and L.K.; visualization, M.E.G and L.K.; supervision, L.K.; project administration, L.K. All authors have read and agreed to the published version of the manuscript.

\subsection{Funding}

The author(s) received no financial support for the research, authorship, and/or publication of this article.

\subsection{Acknowledgements}

The authors would like to thank Mrs. Phyllis Curchack Kornspan for her editorial services.

\subsection{Ethical Approval}

The study was approved by the Ethical Review Board of the Ben Gurion University of the Negev, Israel. All subjects received a detailed explanation of the study goals and provided written consent before participation.

\subsection{Data Availability Statement}

The data presented in this study are available on request from the corresponding author.

\subsection{Competing Interest}

The authors declare that they have no known competing financial interests or personal relationships that could have appeared to influence the work reported in this paper.

\section{References}

[1] Simons, D. G. (1999). Diagnostic Criteria of Myofascial Pain Caused by Trigger Points. Journal of Musculoskeletal Pain, 7(1-2), 111-120. doi:10.1300/j094v07n01_11.

[2] Fernández-de-las-Peñas, C., and Dommerholt, J. (2017). International Consensus on Diagnostic Criteria and Clinical Considerations of Myofascial Trigger Points: A Delphi Study. Pain Medicine, 19(1), 142-150. doi:10.1093/pm/pnx207.

[3] Gerwin, R. D. (2014). Diagnosis of Myofascial Pain Syndrome. Physical Medicine and Rehabilitation Clinics of North America, 25(2), 341-355. doi:10.1016/j.pmr.2014.01.011.

[4] Fricton, J. (2016). Myofascial Pain. Oral and Maxillofacial Surgery Clinics of North America, 28(3), 289-311. doi:10.1016/j.coms.2016.03.010.

[5] Bennett, R. (2007). Myofascial pain syndromes and their evaluation. Best Practice \& Research Clinical Rheumatology, 21(3), 427-445. doi:10.1016/j.berh.2007.02.014. 
[6] Togha, M., Bahrpeyma, F., Jafari, M., \& Nasiri, A. (2020). A sonographic comparison of the effect of dry needling and ischemic compression on the active trigger point of the sternocleidomastoid muscle associated with cervicogenic headache: A randomized trial. Journal of Back and Musculoskeletal Rehabilitation, 33(5), 749-759. doi:10.3233/bmr-171077.

[7] Shah, J. P., Thaker, N., Heimur, J., Aredo, J. V., Sikdar, S., \& Gerber, L. (2015). Myofascial Trigger Points Then and Now: A Historical and Scientific Perspective. PM\&R, 7(7), 746-761. doi:10.1016/j.pmrj.2015.01.024.

[8] Esenyel, M., Caglar, N., \& Aldemir, T. (2000). Treatment of Myofascial Pain. American Journal of Physical Medicine \& Rehabilitation, 79(1), 48-52. doi:10.1097/00002060-200001000-00011.

[9] Alvarez, D. J., \& Rockwell, P. G. (2002). Trigger points: Diagnosis and Management. American Family Physician, $65(4), 653$.

[10] Unalan, H., Majlesi, J., Aydin, F. Y., \& Palamar, D. (2011). Comparison of High-Power Pain Threshold Ultrasound Therapy with Local Injection in the Treatment of Active Myofascial Trigger Points of the Upper Trapezius Muscle. Archives of Physical Medicine and Rehabilitation, 92(4), 657-662. doi:10.1016/j.apmr.2010.11.030.

[11] Draper, D. O., Mahaffey, C., Kaiser, D., Eggett, D., \& Jarmin, J. (2010). Thermal ultrasound decreases tissue stiffness of trigger points in upper trapezius muscles. Physiotherapy Theory and Practice, 26(3), 167-172. doi:10.3109/09593980903423079.

[12] Saggini, R., Di Stefano, A., Carmignano, S. M., Ancona, E., Di Felice, P. A., \& Bellomo, R. G. (2017). The treatment with low frequency acoustic pressure waves (ultrasound diathermy). Therapeutic Considerations and Clinical Reports, 1-6.

[13] Xia, P., Wang, X., Lin, Q., Cheng, K., \& Li, X. (2017). Effectiveness of ultrasound therapy for myofascial pain syndrome: a systematic review and meta-analysis. Journal of Pain Research, Volume 10, 545-555. doi:10.2147/jpr.s131482.

[14] Draper, D. O., Castel, J. C., \& Castel, D. (1995). Rate of Temperature Increase in Human Muscle During $1 \mathrm{MHz}$ and $3 \mathrm{MHz}$ Continuous Ultrasound. Journal of Orthopaedic \& Sports Physical Therapy, 22(4), 142-150. doi:10.2519/jospt.1995.22.4.142.

[15] Watson, T. (2000). The role of electrotherapy in contemporary physiotherapy practice. Manual Therapy, 5(3), $132-141$. doi:10.1054/math.2000.0363.

[16] Watson, T. (2002). Ultrasound dose calculations. Touch, 101(1), 14-17.

[17] Feril, Jr., L. B., \& Kondo, T. (2004). Biological Effects of Low Intensity Ultrasound: The Mechanism Involved, and its Implications on Therapy and on Biosafety of Ultrasound. Journal of Radiation Research, 45(4), 479-489. doi:10.1269/jrr.45.479.

[18] Bradnock, B., Law, H., \& Roscoe, K. (1996). A Quantitative Comparative Assessment of the Immediate Response to High Frequency Ultrasound and Low Frequency Ultrasound ("Longwave Therapy") in the Treatment of Acute Ankle Sprains. Physiotherapy, 82(2), 78-84. doi:10.1016/s0031-9406(05)66955-6.

[19] Meakins, A., \& Watson, T. (2006). Longwave ultrasound and conductive heating increase functional ankle mobility in asymptomatic subjects. Physical Therapy in Sport, 7(2), 74-80. doi:10.1016/j.ptsp.2005.11.006.

[20] Hoch, M. C., Staton, G. S., Medina McKeon, J. M., Mattacola, C. G., \& McKeon, P. O. (2012). Dorsiflexion and dynamic postural control deficits are present in those with chronic ankle instability. Journal of Science and Medicine in Sport, 15(6), 574579. doi:10.1016/j.jsams.2012.02.009.

[21] Hoch, M. C., \& McKeon, P. O. (2010). The Effectiveness of Mobilization with Movement at Improving Dorsiflexion after Ankle Sprain. Journal of Sport Rehabilitation, 19(2), 226-232. doi:10.1123/jsr.19.2.226.

[22] Hall, E. A., \& Docherty, C. L. (2017). Validity of clinical outcome measures to evaluate ankle range of motion during the weightbearing lunge test. Journal of Science and Medicine in Sport, 20(7), 618-621. doi:10.1016/j.jsams.2016.11.001.

[23] Bennell, K., Talbot, R., Wajswelner, H., Techovanich, W., Kelly, D., \& Hall, A. (1998). Intra-rater and inter-rater reliability of a weight-bearing lunge measure of ankle dorsiflexion. Australian Journal of Physiotherapy, 44(3), 175-180. doi:10.1016/s00049514(14)60377-9.

[24] Chisholm, M. D., Birmingham, T. B., Brown, J., MacDermid, J., \& Chesworth, B. M. (2012). Reliability and Validity of a Weight-Bearing Measure of Ankle Dorsiflexion Range of Motion. Physiotherapy Canada, 64(4), 347-355. doi:10.3138/ptc.2011-41.

[25] Farrell, M., \& Richards, J. G. (1986). Analysis of the reliability and validity of the kinetic communicator exercise device. Medicine \& Science in Sports \& Exercise, 18(1), 44-49. doi:10.1249/00005768-198602000-00009.

[26] östenberg, A., Roos, E., Ekdah, C., \& Roos, H. (1998). Isokinetic knee extensor strength and functional performance in healthy female soccer players. Scandinavian Journal of Medicine \& Science in Sports, 8(5), 257-264. doi:10.1111/j.16000838.1998.tb00480.x.

[27] Augustsson, J., \& Thomee, R. (2000). Ability of closed and open kinetic chain tests of muscular strength to assess functional performance. Scandinavian Journal of Medicine and Science in Sports, 10(3), 164-168. doi:10.1034/j.16000838.2000.010003164.x. 
[28] American College of Sports Medicine. (2002). Kraemer WJ, Writing Group Chairman. Position Stand: Progression models in resistance training for healthy adults. Med Sci Sports Exerc, 34, 364-80. doi: 10.1097/00005768-200202000-00027.

[29] Hewett, T. E., Stroupe, A. L., Nance, T. A., \& Noyes, F. R. (1996). Plyometric training in female athletes: decreased impact forces and increased hamstring torques. The American journal of sports medicine, 24(6), 765-773. doi: $10.1177 / 036354659602400611$.

[30] Paterno, M. V., Schmitt, L. C., Ford, K. R., Rauh, M. J., Myer, G. D., Huang, B., \& Hewett, T. E. (2010). Biomechanical Measures during Landing and Postural Stability Predict Second Anterior Cruciate Ligament Injury after Anterior Cruciate Ligament Reconstruction and Return to Sport. The American Journal of Sports Medicine, 38(10), 1968-1978. doi: $10.1177 / 0363546510376053$.

[31] Gustavsson, A., Neeter, C., Thomeé, P., Grävare Silbernagel, K., Augustsson, J., Thomeé, R., \& Karlsson, J. (2006). A test battery for evaluating hop performance in patients with an ACL injury and patients who have undergone ACL reconstruction. Knee Surgery, Sports Traumatology, Arthroscopy, 14(8), 778-788. doi:10.1007/s00167-006-0045-6.

[32] Andrade, M.S., M. Cohen, I.C. Piçarro, and A.C. Silva. "Knee Performance after Anterior Cruciate Ligament Reconstruction." Isokinetics and Exercise Science 10, no. 2 (August 19, 2002): 81-86. doi:10.3233/ies-2002-0088.

[33] Docherty, C., Arnold, B. L., Gansneder, B. M., Hurwitz, S. R., \& Gieck, J. H. (2004). Individuals with Functional Ankle Instability have Contralateral Lowload Force Sense Deficits but not Joint Reposition Sense Deficits. Medicine \& Science in Sports \& Exercise, 36(Supplement), S187. doi:10.1249/00005768-200405001-00896.

[34] Fischer, A. A. (1987). Pressure algometry over normal muscles. Standard values, validity and reproducibility of pressure threshold. Pain, 30(1), 115-126. doi:10.1016/0304-3959(87)90089-3.

[35] Chesterton, L. S., Sim, J., Wright, C. C., \& Foster, N. E. (2007). Interrater Reliability of Algometry in Measuring Pressure Pain Thresholds in Healthy Humans, Using Multiple Raters. The Clinical Journal of Pain, 23(9), 760-766. doi:10.1097/ajp.0b013e318154b6ae.

[36] Kamonseki, D. H., Cedin, L., Tavares-Preto, J., \& Calixtre, L. B. (2018). Reliability, validity, and minimal detectable change of Side Hop Test in male children and adolescents. Physical Therapy in Sport, 34, 141-147. doi:10.1016/j.ptsp.2018.09.009.

[37] Basso, O., \& Pike, J. M. (1998). The Effect of Low Frequency, Long-Wave Ultrasound Therapy on Joint Mobility and Rehabilitation after Wrist Fracture. Journal of Hand Surgery, 23(1), 136-139. doi:10.1016/s0266-7681(98)80248-9.

[38] Ward, A. R., \& Robertson, V. J. (1996). Comparison of Heating of Nonliving Soft Tissue produced by $45 \mathrm{kHz}$ and $1 \mathrm{MHz}$ Frequency Ultrasound Machines. Journal of Orthopaedic \& Sports Physical Therapy, 23(4), $258-266$. doi:10.2519/jospt.1996.23.4.258.

[39] Nakano, J., Yamabayashi, C., Scott, A., \& Reid, W. D. (2012). The effect of heat applied with stretch to increase range of motion: A systematic review. Physical Therapy in Sport, 13(3), 180-188. doi:10.1016/j.ptsp.2011.11.003.

[40] Aguilera, F. J. M., Martín, D. P., Masanet, R. A., Botella, A. C., Soler, L. B., \& Morell, F. B. (2009). Immediate Effect of Ultrasound and Ischemic Compression Techniques for the Treatment of Trapezius Latent Myofascial Trigger Points in Healthy Subjects: A Randomized Controlled Study. Journal of Manipulative and Physiological Therapeutics, 32(7), 515-520. doi:10.1016/j.jmpt.2009.08.001.

[41] Sarrafzadeh, J., Ahmadi, A., \& Yassin, M. (2012). The Effects of Pressure Release, Phonophoresis of Hydrocortisone, and Ultrasound on Upper Trapezius Latent Myofascial Trigger Point. Archives of Physical Medicine and Rehabilitation, 93(1), 7277. doi:10.1016/j.apmr.2011.08.001.

[42] Srbely, J. Z., Dickey, J. P., Lowerison, M., Edwards, M. A., Nolet, P. S., \& Wong, L. L. (2008). Stimulation of myofascial trigger points with ultrasound induces segmental antinociceptive effects: A randomized controlled study. Pain, 139(2), $260-266$. doi:10.1016/j.pain.2008.04.009. 